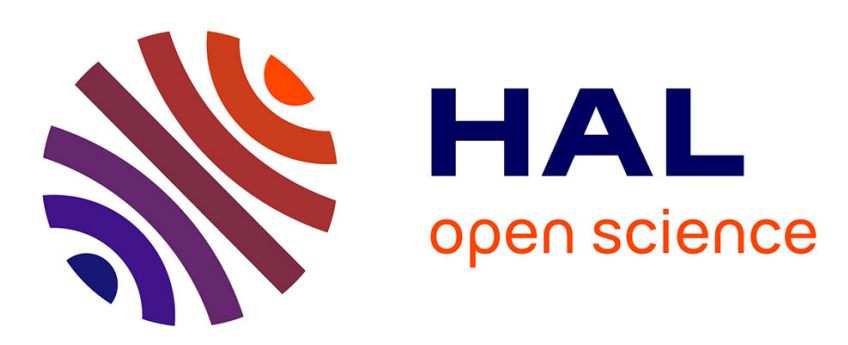

\title{
Resolution of linear viscoelastic equations in the frequency domain using real Helmholtz boundary integral equations
}

Stéphanie Chaillat, Hui Duong Bui

\section{- To cite this version:}

Stéphanie Chaillat, Hui Duong Bui. Resolution of linear viscoelastic equations in the frequency domain using real Helmholtz boundary integral equations. Comptes Rendus Mécanique, 2007, 335, pp.746-750. hal-00178375

\section{HAL Id: hal-00178375 \\ https://hal.science/hal-00178375}

Submitted on 8 Jan 2008

HAL is a multi-disciplinary open access archive for the deposit and dissemination of scientific research documents, whether they are published or not. The documents may come from teaching and research institutions in France or abroad, or from public or private research centers.
L'archive ouverte pluridisciplinaire HAL, est destinée au dépôt et à la diffusion de documents scientifiques de niveau recherche, publiés ou non, émanant des établissements d'enseignement et de recherche français ou étrangers, des laboratoires publics ou privés. 


\title{
Resolution of linear viscoelastic equations in the frequency domain using real Helmholtz boundary integral equations
}

\author{
Stéphanie Chaillat ${ }^{\mathrm{a}, \mathrm{b}}$ Huy Duong Bui ${ }^{\mathrm{a}, \mathrm{c}}$ \\ ${ }^{\mathrm{a}}$ LMS, CNRS UMR 7649, Ecole Polytechnique, 91128 Palaiseau Cedex, France \\ ${ }^{\mathrm{b}}$ LCPC, 58 bd Lefebvre, 75732 Paris Cedex 15, France \\ c Lamsid/EDF/R\&D, 1 av du Général de Gaulle, 92141 Clamart, France \\ Received $* * * * *$; accepted after revision +++++ \\ Presented by Huy Duong Bui
}

\begin{abstract}
Boundary integral equations are well suitable for the analysis of seismic waves propagation in unbounded domains. Formulations in elastodynamics are well developed. In contrast, for the dynamic analysis of viscoelastic media, there are very seldom formulations by boundary integral equations. In this Note, we propose a new and simple formulation of time harmonic viscoelasticity with the Zener model, which reduces to classical elastodynamics if a compatibility condition is satisfied by boundary conditions. Intermediate variables which satisfy the classical elastodynamic equations are introduced. It makes it possible to utilize existing numerical tools of time harmonic elastodynamics. To cite this article: S. Chaillat, H.D. Bui C. R. Mecanique 335 (2007).
\end{abstract}

\section{Résumé}

Résolution des équations de la viscoélasticité linéaire en régime harmonique à l'aide des équations intégrales de frontière de Helmholtz réelles. Les équations intégrales de frontière sont bien adaptées à l'étude de la propagation des ondes sismiques notamment pour les domaines non bornés. Les formulations pour l'élastodynamique sont très développées. Par contre, pour la dynamique des corps viscoélastiques modélisant le sol, peu de formulations par équations intégrales de frontière existent. On propose dans cette Note une nouvelle formulation simple de la viscoélasticité en domaine fréquentiel par un modèle de Zener qui se ramène à la formulation classique de l'élastodynamique, si les conditions à la frontière respectent une condition de compatibilité. Des variables intermédiaires qui respectent les équations de l'élastodynamique classique sont introduites. Il est alors possible de réutiliser les outils numériques existant pour l'élastodynamique harmonique. Pour citer cet article : S. Chaillat, H.D. Bui C. R. Mecanique 335 (2007).

Key words: Computational solid mechanics ; Viscoelasticity ; Boundary integral equations

Mots-clés : Mécanique des solides numérique; Viscoélasticité; Équations intégrales de frontière 


\section{Version française abrégée}

Les équations intégrales de frontière sont bien adaptées à l'étude de la propagation des ondes sismiques dans les domaines non bornés. Les formulations en élastodynamique sont bien développées [1]. D'un autre côté, il y a très peu de formulations équations intégrales de frontière pour l'étude dynamique des milieux viscoélastiques. La méthode classique consiste à utiliser des coefficients de Lamé complexes [9]. Dans cette Note, on propose une nouvelle méthode simple pour la viscoélasticité en domaine fréquentiel, qui se ramène à l'élastodynamique classique par un simple changement de variables. Le principal avantage de cette méthode est de conserver des constantes élastiques réelles et donc de permettre de réutiliser les outils numériques existant pour l'élastodynamique en domain fréquentiel [12].

Le modèle rhéologique utilisé dans cette Note est un modèle de Zener (Fig. 1). L'idée principale est de chercher le déplacement sous la forme découplée mais restrictive (1). Ensuite, les notations introduites dans [11] sont utilisées (2). Ces variables intermédiaires vérifient les lois de l'élasticité. En introduisant l'angle $\psi$ tel que $\tan \psi=\alpha \omega$, on montre (3) que les variables $\boldsymbol{u}^{*}$ et $\boldsymbol{u}$ sont déphasées de $\psi$ mais ont la même pulsation $\omega$.

On note de la même manière l'angle $\phi$ tel que $\tan \phi=\beta \omega$. En utilisant le fait que les variables $\boldsymbol{u}^{*}$ et $\boldsymbol{\sigma}^{*}$ doivent être en phase, on montre que $\boldsymbol{u}$ et $\boldsymbol{\sigma}$ doivent être déphasées de $\theta=\psi-\phi$ (4). De plus, pour que $\boldsymbol{u}^{*}$ et $\boldsymbol{\sigma}^{*}$ puissent satisfaire l'équation de la dynamique (5), $\theta$ doit être petit. Ceci revient à ajouter l'hypothèse $\omega \ll \omega_{1}=1 /|\alpha-\beta|$.

Supposons que les données $\boldsymbol{u}^{d}$ sur $\partial \Omega_{1}$ et $\boldsymbol{\sigma}^{d}$. $\boldsymbol{n}$ sur $\partial \Omega_{2}$ soient compatibles sur la frontière (afin de pouvoir noter $\boldsymbol{u}$ sous la forme (1), donc déphasées de $\theta=\psi-\phi$ ). Par exemple, les données suivantes sont compatibles :

(i) $\boldsymbol{u}^{d} \neq 0$ sur $\partial \Omega_{1}$ et $\boldsymbol{\sigma}^{d} \cdot \boldsymbol{n}=0$ sur $\partial \Omega_{2}$

(ii) $\boldsymbol{u}^{d}=0$ sur $\partial \Omega_{1}$ et $\boldsymbol{\sigma}^{d} \cdot \boldsymbol{n} \neq 0$ sur $\partial \Omega_{2}$

Ainsi les variables $\boldsymbol{u}^{*}$ et $\boldsymbol{\sigma}^{*}$ qui s'en déduisent sont en phase. La solution du problème visco-élastique linéaire est alors obtenue en résolvant l'équation intégrale classique de l'élastodynamique pour $\boldsymbol{u}^{*}$ et $\boldsymbol{\sigma}^{*}$. Connaissant la solution $\boldsymbol{u}^{*}$ (resp. $\boldsymbol{\sigma}^{*}$ ), on peut calculer sans difficulté $\boldsymbol{u}$ (resp. $\boldsymbol{\sigma}$ ). En effet, les solutions du problème élastodynamique $\boldsymbol{u}^{*}$ et $\boldsymbol{\sigma}^{*}$ ont respectivement une amplitude égale à $\frac{v(x)}{\cos \psi}$ et $\frac{w(x)}{\cos \phi}$ (d'après (3) et (4)). Pour connaître l'amplitude de $\boldsymbol{u}$ (resp. $\boldsymbol{\sigma}$ ), il faut donc multiplier l'amplitude de $\boldsymbol{u}^{*}$ (resp. $\boldsymbol{\sigma}^{*}$ ) par $\cos \psi($ resp. $\cos \phi)$ où $\psi=\tan ^{-1} \alpha \omega$ et $\phi=\tan ^{-1} \beta \omega$.

En pratique, la résolution numérique de cette formulation peut être effectuée en utilisant les travaux effectués en élastodynamique [14].

\section{Introduction}

The main advantage of boundary element method (BEM) is that only the domain boundary is discretized. As a result, the method is well suitable for the study of problems in unbounded domains. So, the boundary integral formulation of linear elasticity is used to study seismic wave propagation [1].

But, the ideal model of a linear elastic soil is not adapted in a lot of cases. It is necessary to take into account the soil damping factor and so to use a formulation for dynamic viscoelasticity.

In time domain, various methods have been proposed for BEM formulation of viscoelasticity [2]. They are sorted into three kinds. The first formulation is developed by applying the elastic-viscoelastic cor-

Email addresses: chaillat@lms.polytechnique.fr (corresponding author) (Stéphanie Chaillat), hdb@lms .polytechnique.fr (Huy Duong Bui). 
respondence principle. An integral transform (according to time) is performed on the boundary integral equation of elastodynamics. Generally, Laplace transform is considered, for example in the works of Rizzo [3] or Kusama [4]. Then, the viscoelastic fundamental solutions are obtained by applying the elasticviscoelastic correspondence principle to elastodynamic solutions. Various works deal with the reduction of the last step: the back transform to time domain.

The second class of methods uses the fundamental solutions of elastodynamics in time domain. Once the convolution with time shape functions is integrated analytically, the equation is transformed in Laplace domain where the correspondence principle is applied. Then, a numerical inverse transformation is required [5] to lead to a time domain boundary element formulation. Various works use the "convolution quadrature method" developed by Lubich [6] to evaluate the convolution [7].

The last class of methods directly requires the knowledge of the viscoelastic fundamental solutions in time domain. Using differential systems of Kelvin and Boltzmann models, Mesquita [8] determines integral formulations adapted to each model. With those methods, only for the simplest viscoelastic models, the fundamental solutions are available analytically and one does not consider works in elastodynamics.

In frequency domain, the usual method is to replace the Lamé's constants by complex values [9].

In this Note, a simple method to formulate the boundary integral equations for viscoelasticity, with a Zener model, is presented. This method, based on the introduction of new intermediate variables, reuses the classical formulation of elastodynamics and presents the advantage to keep real values of Lamé's constants. To the authors knowledge, a formulation similar to ours does not exist in the literature.

\section{Rheological model}

Various rheological models exist to model the viscoelastic behavior of a material [10]. The KelvinVoigt model is well adapted to model solids. The Maxwell model is in general used to model fluids. The rheological model used in this Note is a Zener with a dashpot impedance $\eta$ and elastic constants $k_{0}$ and $k_{1}$ (Fig 1).

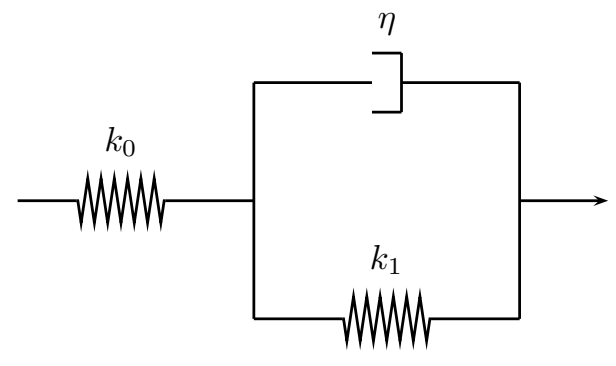

Figure 1. Zener model

In this model, if $\dot{\epsilon}=0$, so the dashpot has not effect, it is called a "delayed modulus". The model is equivalent to two springs connected in series. On the contrary, if $\dot{\epsilon}=\infty$, the dashpot does not have time to react, it is called an "instantaneous modulus".

\section{Formulation of the problem}

In the following, usual typeface letters denote scalar quantities while boldface letters denote vectors, matrices or tensors. The partial derivate is denoted using a comma $\left(\frac{\partial f}{\partial x}=f_{, x}\right)$. 
The study is made in the frequency domain. The main idea is to not define the displacement in the classical form $\boldsymbol{u}(\boldsymbol{x}, t)=\boldsymbol{u}(\boldsymbol{x}) e^{i \omega t}$ ( $\omega$ denoting the circular frequency) but under the restictive condition:

$$
\boldsymbol{u}(\boldsymbol{x}, t)=\boldsymbol{v}(\boldsymbol{x}) \cos \omega t
$$

where $\boldsymbol{v}(\boldsymbol{x})$ is a real. As a result, the variables $\boldsymbol{x}$ and $t$ are uncoupled.

The three-dimensional generalization of the Zener constitutive law has been proposed by I. Goriacheva in [11] and is used in the present Note, denoting $\boldsymbol{\sigma}$ the stress tensor and $\boldsymbol{\epsilon}$ the strain tensor:

$$
\left\{\begin{array}{l}
\boldsymbol{\sigma}^{*}=\boldsymbol{\sigma}+\beta \frac{\partial \boldsymbol{\sigma}}{\partial t} \\
\boldsymbol{\epsilon}^{*}=\boldsymbol{\epsilon}+\alpha \frac{\partial \boldsymbol{\epsilon}}{\partial t} \\
\boldsymbol{u}^{*}=\boldsymbol{u}+\alpha \frac{\partial \boldsymbol{u}}{\partial t}
\end{array}\right.
$$

The coefficients $\alpha$ and $\beta(\alpha>\beta)$ are determined by:

$$
\alpha=\frac{\eta}{k_{1}}, \beta=\frac{\eta}{k_{0}+k_{1}}
$$

The tensors $\boldsymbol{\sigma}^{*}$ and $\boldsymbol{\epsilon}^{*}$ are linked by the constitutive equation of isotropic linear elasticity, with the Lamé's coefficients $\lambda$ and $\mu$ of the delayed modulus, $\boldsymbol{\sigma}^{*}=L \boldsymbol{\epsilon}^{*}$.

With notation (1), we note that $\boldsymbol{u}(\boldsymbol{x}, t)$ and $\dot{\boldsymbol{u}}(\boldsymbol{x}, t)$ are dephased of $\pi / 2$. Using notation (2), we obtain $\boldsymbol{u}^{*}(\boldsymbol{x}, t)=\boldsymbol{v}(\boldsymbol{x})[\cos \omega t-\alpha \omega \sin \omega t]$. Noting the angle $\psi$ such that $\tan \psi=\alpha \omega(0 \leq \psi<\pi / 2$, i.e. $\cos \psi \neq 0)$, it follows:

$$
\boldsymbol{u}^{*}(\boldsymbol{x}, t)=\frac{\boldsymbol{v}(\boldsymbol{x})}{\cos \psi} \cos (\omega t+\psi)
$$

It is now clear that the variables $\boldsymbol{u}^{*}$ and $\boldsymbol{u}$ are dephased of $\psi$ but have the same circular frequency $\omega$.

Then, noting $\boldsymbol{\sigma}(\boldsymbol{x}, t)=\boldsymbol{w}(\boldsymbol{x}) \cos (\omega t+\theta)$, we obtain $\boldsymbol{\sigma}^{*}(\boldsymbol{x}, t)=\boldsymbol{w}(\boldsymbol{x})[\cos (\omega t+\theta)-\beta \omega \sin (\omega t+\theta)]$. Defining in the same way that for $\boldsymbol{u}$, the angle $\phi$ such that $\tan \phi=\beta \omega(0 \leq \phi<\pi / 2$, i.e. $\cos \phi \neq 0)$, it follows:

$$
\boldsymbol{\sigma}^{*}(\boldsymbol{x}, t)=\frac{\boldsymbol{w}(\boldsymbol{x})}{\cos \phi} \cos (\omega t+\phi+\theta)
$$

But, the variables $\boldsymbol{\sigma}^{*}$ and $\boldsymbol{u}^{*}$ are known to satisfy the linear elasticity equations, as a result they have to be in phase. It follows that $\psi=\phi+\theta$. Finally, $\boldsymbol{\sigma}$ and $\boldsymbol{u}$ have to be dephased of $\theta=\psi-\phi$.

\section{Boundary integral formulation}

The boundary integral equation method for this formulation of viscoelasticity is now defined using the well-known method for elastodynamics. In fact, the main advantage of this formulation is that only a simple change of variables is introduced.

Boundary integral equation. The quantities $\boldsymbol{\sigma}^{*}$ and $\boldsymbol{u}^{*}$ which are linked by the elastic law, are now shown to satisfy the dynamic equation. $\boldsymbol{\sigma}$ and $\boldsymbol{u}$ can satisfy the dynamic equation $\operatorname{div} \boldsymbol{\sigma}-\rho \ddot{\boldsymbol{u}} \cong 0$ if and only if $\boldsymbol{\sigma}$ and $\boldsymbol{u}$ are almost in phase, that is to say if and only if the angle $\theta$ is small. The difference introduced by the dephasing between $\boldsymbol{\sigma}$ and $\boldsymbol{u}$, in the dynamic equation, is proportional to $\rho \omega^{2} \theta$. It can be easily proved that $\theta \cong|\alpha-\beta| \omega$. As a result, $\rho \omega^{2} \theta$ is proportional to $\rho \omega^{3}|\alpha-\beta|$ and we remark that we have $\operatorname{div} \boldsymbol{\sigma}-\rho \ddot{\boldsymbol{u}} \cong 0$ to order $O\left(\omega^{3}\right)$. It follows that: 


$$
\operatorname{div} \boldsymbol{\sigma}^{*}-\rho \ddot{\boldsymbol{u}}^{*} \equiv(\operatorname{div} \boldsymbol{\sigma}-\rho \ddot{\boldsymbol{u}})+\beta(\operatorname{div} \dot{\boldsymbol{\sigma}}-\rho \dddot{\boldsymbol{u}})+(\beta-\alpha) \rho \dddot{\boldsymbol{u}} \cong(\beta-\alpha) \rho \dddot{\boldsymbol{u}}
$$

Using the definition of $\boldsymbol{u}(1)$, we obtain that $\operatorname{div} \boldsymbol{\sigma}^{*}-\rho \ddot{\boldsymbol{u}}^{*} \cong 0$ and that the difference introduced in this dynamic equation is equally proportional to $\rho \omega^{3}|\alpha-\beta|$ if and only if:

$$
|\alpha-\beta| \omega \ll 1 \quad \text { that is to say } \quad|\theta| \ll 1 .
$$

As a result, if $\omega$ is much less than the limit frequency $\omega_{1}=1 /|\alpha-\beta|$, the notation (1) is compatible with elastodynamics.

Since $|\alpha-\beta|$ is proportional to the viscosity coefficient $\eta$ (for a given set of elastic constant $k_{0}$ and $k_{1}$ ), the lower the coefficient $\eta$, the higher the limit frequency $\omega_{1}$ (in soil mechanics $\eta$ is small so that $\omega_{1}$ is very large). Thus the hypothesis of "low" frequency $\omega \ll \omega_{1}$ (including the quasi-static case $\omega=0$ ), which we suppose in the following, is satisfed in soil mechanics, for a large frequency range. This leads to the boundary integral formulation which is the same that for elastodynamics: $\operatorname{div} \boldsymbol{\sigma}^{*}+\rho \omega^{2} \boldsymbol{u}^{*}=0$, $\sigma^{*}=L \epsilon^{*}$.

This formulation is now recalled [12]. The stress vector $\boldsymbol{T}^{\boldsymbol{n}} \boldsymbol{u}$ on a plane of normal $\boldsymbol{n}$ is defined by the operator ( $\lambda$ and $\mu$ representing the Lamé's constants): $\boldsymbol{T}^{\boldsymbol{n}}=2 \mu \frac{\partial}{\partial n}+\lambda \boldsymbol{n} . \boldsymbol{d i v}+\mu \boldsymbol{n} \wedge \boldsymbol{r o t}$.

Noting $\Omega$ the region of space occupied by an elastic solid with isotropic constitutive properties, the displacement $\boldsymbol{u}$ at an interior point $\boldsymbol{x} \in \Omega$ is given by:

$$
u_{k}(\boldsymbol{x})=\int_{\partial \Omega}\left[\left(\boldsymbol{T}^{\boldsymbol{n}} \boldsymbol{u}(\boldsymbol{y})\right)_{i} U_{i}^{k}(\boldsymbol{x}, \boldsymbol{y} ; \omega)-u_{i}(\boldsymbol{y})\left(\boldsymbol{T}^{n} \boldsymbol{U}^{\boldsymbol{k}}(\boldsymbol{x}, \boldsymbol{y} ; \omega)\right)_{i}\right] d S_{y} \quad(\boldsymbol{x} \in \Omega),
$$

where $U_{i}^{k}(\boldsymbol{x}, \boldsymbol{y} ; \omega)$ denotes the $i$-th component of the elastodynamic fundamental solution, in the frequency domain, for an infinite space.

When $\boldsymbol{x} \in \partial \Omega$, a singularity occurs in $\boldsymbol{y}=\boldsymbol{x}$. With the help of a well-documented limiting process, equation (7) yields the integral equation:

$$
c_{i k}(\boldsymbol{x}) u_{i}(\boldsymbol{x})=(P . V .) \int_{\partial \Omega}\left[\left(\boldsymbol{T}^{\boldsymbol{n}} \boldsymbol{u}(\boldsymbol{y})\right)_{i} U_{i}^{k}(\boldsymbol{x}, \boldsymbol{y} ; \omega)-u_{i}(\boldsymbol{y})\left(\boldsymbol{T}^{\boldsymbol{n}} \boldsymbol{U}^{\boldsymbol{k}}(\boldsymbol{x}, \boldsymbol{y} ; \omega)\right)_{i}\right] d S_{y} \quad(\boldsymbol{x} \in \partial \Omega),
$$

where (P.V.) $\int$ indicates a Cauchy principal value (CPV) singular integral and the free term $c_{i k}(\boldsymbol{x})$ is equal to $0.5 \delta_{i k}$ in the usual case where $\partial \Omega$ is smooth at $\boldsymbol{x}$.

Boundary conditions. We consider a domain $\Omega$, of boundary $\partial \Omega$ on which mixed but independent boundary conditions are imposed $\left(\partial \Omega=\partial \Omega_{1}+\partial \Omega_{2}\right.$ and $\left.\partial \Omega_{1} \bigcap \partial \Omega_{2}=\emptyset\right)$. On $\partial \Omega_{1}$, the imposed displacement is noted as in (1):

$$
\boldsymbol{u}^{d}(\boldsymbol{x}, t)=\boldsymbol{u}^{d}(\boldsymbol{x}) \cos \omega t
$$

on $\partial \Omega_{2}$, the stress vector is written in the same manner:

$$
\boldsymbol{t}^{d}(\boldsymbol{x}, t)=\boldsymbol{w}^{d}(\boldsymbol{x}) \cos \omega(t+\theta),
$$

Assume that the data $\boldsymbol{u}^{d}$ on $\partial \Omega_{1}$ and $\boldsymbol{\sigma}^{d} \cdot \boldsymbol{n}$ on $\partial \Omega_{2}$ are compatible (if $\boldsymbol{u}$ can be defined in form (1) so the variables are dephased of $\theta=\psi-\phi)$. For example, the following data are compatible:

(i) $\boldsymbol{u}^{d} \neq 0$ (circular frequency $\omega$ ) on $\partial \Omega_{1}$ and $\boldsymbol{\sigma}^{d} \cdot \boldsymbol{n}=0$ on $\partial \Omega_{2}$

(ii) $\boldsymbol{u}^{d}=0$ on $\partial \Omega_{1}$ and $\boldsymbol{\sigma}^{d} \cdot \boldsymbol{n} \neq 0$ (circular frequency $\omega$ ) on $\partial \Omega_{2}$

As a result, the intermediate variables $\boldsymbol{u}^{*}$ and $\boldsymbol{\sigma}^{*}$, are necessary in phase. The problem in $\boldsymbol{u}^{*}$ and $\sigma^{*}$ is solved using the well-known boundary integral formulation of elastodynamics in the frequency domain. Having the solution $\boldsymbol{u}^{*}$ (resp. $\boldsymbol{\sigma}^{*}$ ), $\boldsymbol{u}$ (resp. $\boldsymbol{\sigma}$ ) is easily computed. Indeed, the solutions of the elastodynamic problem $\boldsymbol{u}^{*}$ and $\boldsymbol{\sigma}^{*}$ have respectively an amplitude equal to $\frac{v(x)}{\cos \psi}$ and $\frac{w(x)}{\cos \phi}((3)$ and (4)). 
So, to compute the amplitude of $\boldsymbol{u}$ (resp. $\boldsymbol{\sigma}$ ), one only has to multiply the amplitude of $\boldsymbol{u}^{*}$ (resp. $\boldsymbol{\sigma}^{*}$ ) by $\cos \psi($ resp. $\cos \phi)$ where $\psi=\tan ^{-1} \alpha \omega$ and $\phi=\tan ^{-1} \beta \omega$.

\section{Conclusion}

A new and simple formulation of time harmonic viscoelasticity (including the quasi-static case) have been presented. Hence, it has been shown that this problem reduces to a classical elastic problem by a simple change of variables if the boundary conditions respect a restrictive condition. It makes possible to reuse existing numerical tools of time harmonic elastodynamics. The speed up of the computation can be done using the Fast multipole method [14].

\section{References}

[1] J.F. Semblat, A.M. Duval, P. Dangla. Seismic site effects in deep alluvial basin : numerical analysis by boundary element method Computers and geotechnics 29 (2002) 573-585.

[2] M. Schanz. Wave Propagation in Viscoelastic and Poroelastic Continua, Springer, 2001.

[3] F.J. Rizzo and D.J. Shippy. An application of the correspondence principle of linear viscoelasticity theory SIAM J. Appl. Math. 21 (1971) 321-330.

[4] T. Kusama and Y. Mitsui. Boundary element method applied to linear viscoelastic analysis. Appl. Math. Modelling 6 (1982) 285-290.

[5] L. Gaul and M. Schanz. Dynamics of viscoelastic solides treated by boundary element approaches in time domain Eur. J. Mech. A/Solides 13 (1994) 43-59.

[6] C. Lubich. Convolution quadrature and discretized operational calculus, I Numerische Mathematik 52 (1988) $129-145$.

[7] M. Schanz, H. Antes. A new visco- and elastodynamic time domain boundary element formulation Computational Mechanics 20 (1997) 452-459.

[8] A.D. Mesquita, H.B. Coda. A simple Kelvin and Boltzmann viscoelastic analysis of three-dimensional solids by boundary element method Engineering analysis with boundary elements 27 (2003) 885-895.

[9] J. Dominguez Boundary Elements in Dynamics International Series on Computational Engineering, Computational Mechanics, Southampton; Elsevier Applied Science, London, UK, 1993.

[10] W. Flügge. Viscoelasticity, Springer-Verlag, 1975.

[11] I.G. Goriacheva. Contact problem of rolling of a viscoelastic cylinder on a base of the same material PMM 37 (1973) 925-933.

[12] M. Bonnet. Boundary integral equation method for solids and fluids, Wiley, 1999.

[13] A.C. Eringen and E.S. Suhubi. Elastodynamics, Academic Press,II-linear theory, 1975.

[14] S. Chaillat, M. Bonnet, J.F. Semblat. A Fast Multipole Method formulation for 3D elastodynamics in the frequency domain C. R. Mecanique 335 (2007), accepted. 